\title{
Teleconsulta en la pandemia por Coronavirus: desafíos para la telemedicina pos-COVID-19
}

\author{
Teleconsultation in a pandemic due to Coronavirus: challenges for \\ telemedicine in the post-COVID-19 era
}

Juan Ricardo Márquez V., MD*

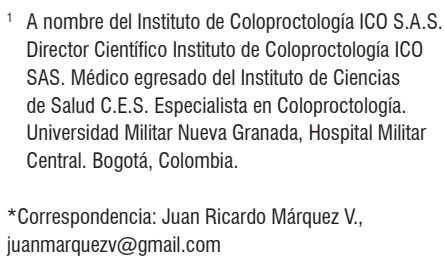

A nombre del Instituto de Coloproctología ICO S.A.S. Director Científico Instituto de Coloproctología ICO SAS. Médico egresado del Instituto de Ciencias de Salud C.E.S. Especialista en Coloproctología. Universidad Militar Nueva Granada, Hospital Militar Central. Bogotá, Colombia.

*Correspondencia: Juan Ricardo Márquez V., juanmarquezv@gmail.com

Fecha recibido: $22 / 04 / 20$ Fecha aceptado: $27 / 04 / 20$

\begin{abstract}
Resumen
Con el objetivo de detener la transmisión del coronavirus (declarado como pandemia por la Organización Mundial de la Salud [OMS]), y prevenir la propagación de la enfermedad COVID-19, el Gobierno de Colombia decretó un período de aislamiento social y confinamiento obligatorio en el territorio nacional a partir del 24 de marzo de 2020. El Instituto de Coloproctología (ICO) diseñó un programa de teleconsulta, junto con la entidad prestadora de los servicios de salud (EPS) SURA, apoyado en las tecnologías de la información disponibles. Se buscó mantener las actividades propias de la coloproctología y la fisioterapia del piso pélvico para garantizar a los pacientes la atención y el acceso ininterrumpido. Luego de 25 días, se programaron 626 consultas (coloproctología $62 \%$ y fisioterapia del piso pélvico $38 \%$ ) con un porcentaje de ejecución del 94 \% y una resolutividad del $78 \%$. Estas cifras demuestran que la telemedicina, al igual que otras actividades basadas en el teletrabajo, llegó para quedarse y trae consigo altos niveles de satisfacción para médicos, pacientes y entidades prestadoras de los servicios de salud. En este campo, también, el mundo nunca será el mismo.
\end{abstract}

\section{Palabras clave}

COVID-19, pandemia, coronavirus, teleconsulta, telemedicina, telesalud.

\begin{abstract}
After being decreed, as of March 24, 2020, social isolation and mandatory confinement began in the national territory with the aim of stopping the transmission and preventing the spread of the coronavirus as it was declared a pandemic by the World Organization of Health (WHO). The Instituto de Coloproctología (ICO) designed and developed, together with its largest insurance company EPS SURA and through the available technologies of information, a teleconsultation program, with the aim of maintaining the activities of colorectal surgery and pelvic floor biofeedback and thus guaranteeing uninterrupted medical care and access for our patients. After twenty-five days, 626 consultations were scheduled (colorectal surgery $62 \%$ and pelvic floor biofeedback $38 \%$ ) with a percentage of execution of $94 \%$ and a resolution of $78 \%$. This shows that telemedicine, like all teleworking activities, came to stay with high levels of satisfaction, in a world that will never be the same again.
\end{abstract}

\section{Key words}

COVID-19, pandemic, coronavirus, teleconsultation, telemedicine, telehealth. 
el país, y el 11 del mismo mes la Organización Mundial de la Salud (OMS) declaró el actual brote de la enfermedad como una pandemia. La OMS solicitó a los países la adopción de medidas prematuras para detener la transmisión y prevenir la propagación del virus.

Colombia, en consecuencia, emitió ese decreto, lo que determinó el aislamiento y el distanciamiento social mediante una cuarentena obligatoria de 20 días, que se amplió posteriormente hasta el 11 de mayo. Luego de determinar un aumento sostenido de nuevos casos por coronavirus, y ante la necesidad insatisfecha de acceso a los servicios básicos de salud, se reglamentó el Concepto Clínico Especializado (Asesoría Clínica Virtual) aplicado como un servicio de telemedicina.

El 12 de abril de 2020, el presidente y sus ministros firmaron el decreto 538, amparado entre otros en:

- La Ley 1419 de 2010, "por la cual se establecen los lineamientos para el desarrollo de la Telesalud en Colombia".

- La Resolución 2003 de 2014 que define el servicio de telemedicina (dentro del grupo: otros servicios), y contempla en sus diferentes modalidades la modalidad telemedicina, con sus estándares y criterios de cumplimiento.

En el artículo 8, parágrafos 1 y 2 de dicha resolución, reza:

"Durante el término de la emergencia sanitaria declarada por el Ministerio de Salud y Protección Social, con ocasión de la pandemia derivada del Coronavirus
COVID-19, y con el fin de facilitar el acceso a los servicios de salud, los prestadores servicios de salud deberán implementar plataformas digitales accesibles con estándares básicos de audio y video que permitan el diagnóstico y seguimiento del paciente".

Estos actos administrativos definen el alcance de la telesalud, determinan la telemedicina como una modalidad, y reglamentan la atención en esta modalidad. Por lo anterior, y ante la ampliación de la cuarentena hasta el 11 de mayo, la salud y amplios sectores de la economía se han visto en el reto de reinventarse. Gracias a la capacidad resiliente y de innovación, pero también bajo el amparo del marco legal anotado, la reinvención se apalanca, como se afirma, en que toda crisis es necesaria para que la humanidad avance, pues es en esos momentos surgen las grandes transformaciones.

\section{MATERIALES Y MÉTODOS}

Este reporte describe una experiencia sobre la implementación de un servicio de teleconsulta en una institución de servicios médico-quirúrgicos. Para su ejecución, se diseñó una estrategia juntamente con la entidad prestadora de los servicios de salud (EPS) SURA (que integra la teleconsulta en sus operaciones y tiene codificadas las actividades que conforman el proceso) (Figura 1). Ese protocolo guía las actividades del Instituto de Coloproctología (ICO) S.A.S. para las actividades de teleconsulta realizada desde el domi-

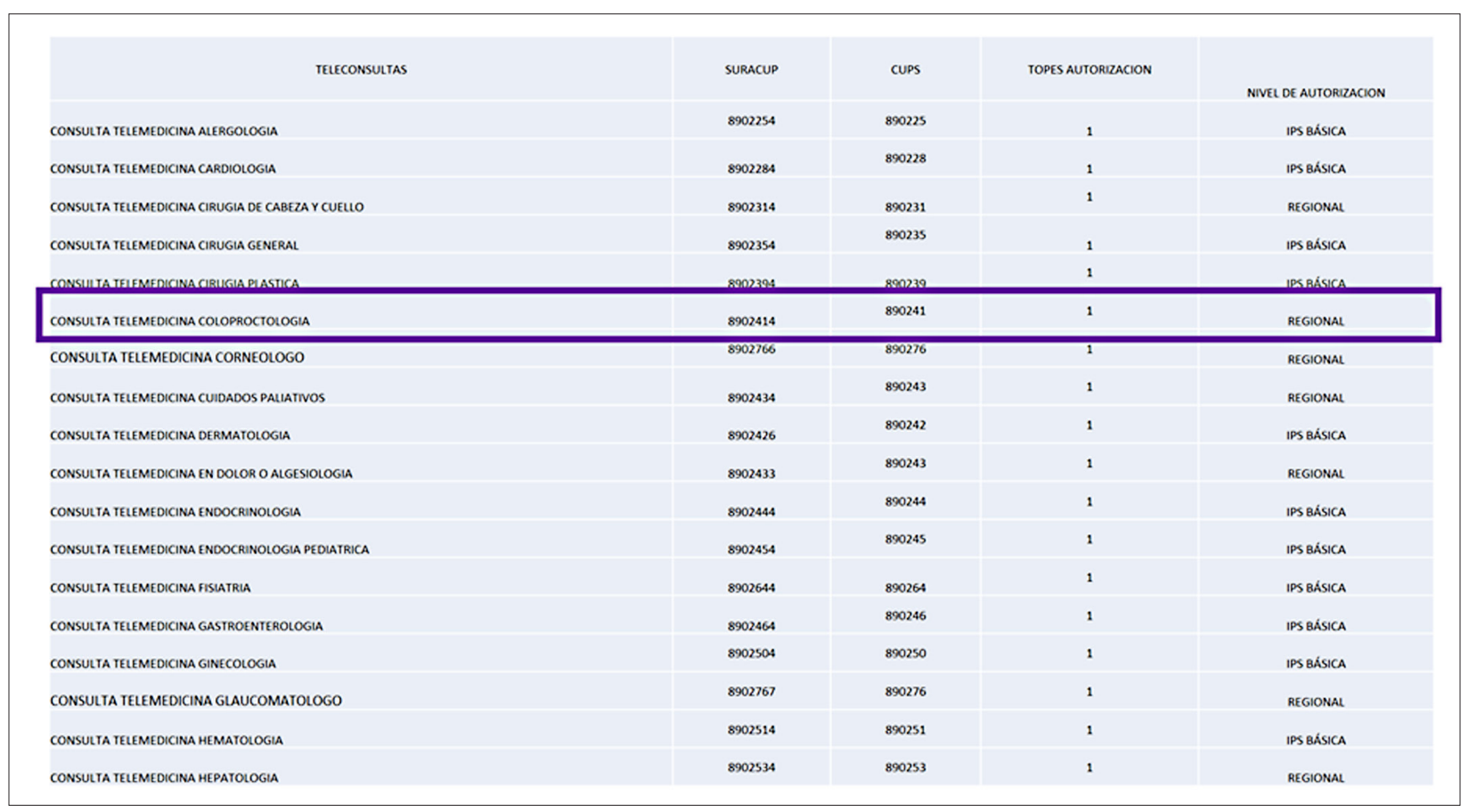

Figura 1. Codificación de especialidades de la EPS SURA para la teleconsulta. Fuente: EPS-SURA. 
cilio de cada especialista. La entidad dispuso su plataforma virtual de historia clínica electrónica (IPSA -Agenda Web) (Figura 2); una estructura robusta que reúne la información clínica de sus afiliados bajo los estándares de seguridad e inviolabilidad requeridos para la historia clínica electrónica, que está ubicada en el sitio sunube.com (Figura 3).

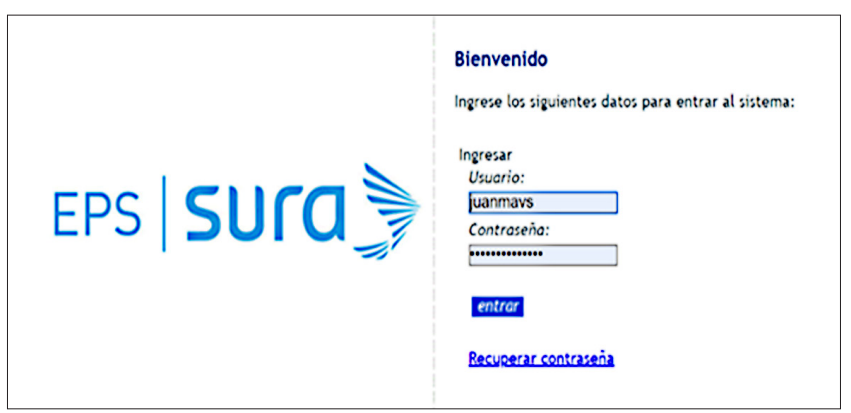

Figura 2. Portal página IPSA - SURA WEB. Fuente: EPS SURA.

Para responder a las exigencias de la medida de aislamiento social obligatorio o cuarentena, se decidió que el personal de ICO cumpliera sus funciones desde sus residencias. Tal decisión implicó un apoyo logístico para las secretarias, a quienes se les proveyó una conexión confiable a internet, una terminal de computador y el redireccionamiento de teléfonos IP del instituto a su computador. Utilizamos la aplicación Trello ${ }^{\circledast}$ (un software de administración de proyectos con interfaz web) que, en la práctica, es un tablero virtual que integra ideas, tareas, imágenes o enlaces. Es una aplicación versátil y fácil de usar para la programación y seguimiento de cualquier tipo de proyecto o tarea que implique organizar información (Figura 4).

El proceso señala que las secretarias se comunican con el paciente, y les explican que, dada la situación actual de contingencia y con su aceptación, se les hará la consulta de manera telefónica; adicionalmente, también con su aceptación, se les instruye para la toma de fotografías a los resultados de laboratorio o a los exámenes relevantes, y se les pide que las envíen a un número de WhatsApp en el que, a su vez, una funcionaria de ICO las recibe y las sube a la bandeja de entrada de Trello ${ }^{\oplus}$. A los especialistas se les sugirió conseguir tarjetas SIM independientes para sus teléfonos celulares para atender esta actividad.

El programa comenzó a operar el 24 de marzo ofreciendo las actividades de coloproctología y fisioterapia de piso pélvico. En ambos servicios, el especialista se comunica con el paciente, le informa y se anota, de manera rutinaria, en la historia clínica de las plataformas bajo el decreto 538 del 12 de abril de 2020 que reza: "durante el término de la emergencia sanitaria declarada por el Ministerio de Salud y Protección Social, con ocasión de la pandemia derivada del Coronavirus COVID-19, y con el fin de facilitar el acceso a los servicios de salud, los prestadores de servicios de salud deberán implementar plataformas digitales accesibles con estándares básicos de audio y video que permitan el diagnóstico y seguimiento del paciente y estando en un estado de cuarentena obligatoria y previa aceptación por parte del paciente esta consulta se hace de manera virtual".

Cumplido este protocolo, se dialoga y se interroga al paciente. En caso de que sea su primera consulta, se elabora el ingreso y se diligencia la historia clínica. Si se trata de un control, se confirma y se registran la evolución, la efectividad del tratamiento actual y, en caso de tratarse de una fisioterapia, se hace el seguimiento a los ejercicios, videos y tareas de las actividades previamente ordenadas. Igualmente, se revisan los exámenes enviados con anterioridad, se aclaran dudas, se ofrecen recomendaciones e instrucciones, se ordenan exámenes pertinentes y, si es del caso, se formula un tratamiento.

\section{RESULTADOS}

Transcurridos 25 días del proceso, con fecha de corte al 17 de abril de 2020, se registraron 626 consultas (coloproctología $62 \%$ y fisioterapia del piso pélvico $38 \%$ ). Efectivamente, fueron respondidas y atendidas 588 consultas, lo que implica una ejecución del $94 \%$, con una resolutividad del $78 \%$ (programación de cirugías, plan de ejecución de ejercicios pélvicos, orden de exámenes y formulación, incluida la elaboración de Mipres y Altas).

Cuando los contactos se dividieron según el tipo de patología, se identificó la siguiente atención: colorrectal, se atendió patología anorrectal (45\%); patología colónica (médico $10 \%$ y quirúrgica $10 \%$ ); y consulta de enfermedad inflamatoria intestinal (EII) (35\%).

Cabe aclarar que se han programado para cirugía solamente pacientes con patología anorrectal. Aquellos pacientes con patología colorrectal quirúrgica se han aplazado temporalmente con manejo médico, con instrucciones precisas de que, ante un eventual agravamiento de su condición, es preciso consultar de manera urgente a la clínica. El cuerpo médico es ferviente defensor del examen físico, especialmente anorrectal, y solo se han programado casos muy evidentes con la advertencia de que, al llegar a preadmisiones de la clínica, el día de la cirugía se repetirá la revisión con el objetivo de confirmar el diagnóstico que llevó a programar la operación, y proceder a la diligencia y firma conjunta del consentimiento informado.

\section{DISCUSIÓN}

Periódicamente, el mundo enfrenta epidemias y, en menor número, pandemias. La más reciente y recordada ocurrió a inicios del siglo XX, en 1918, al terminar la Primera Guerra 


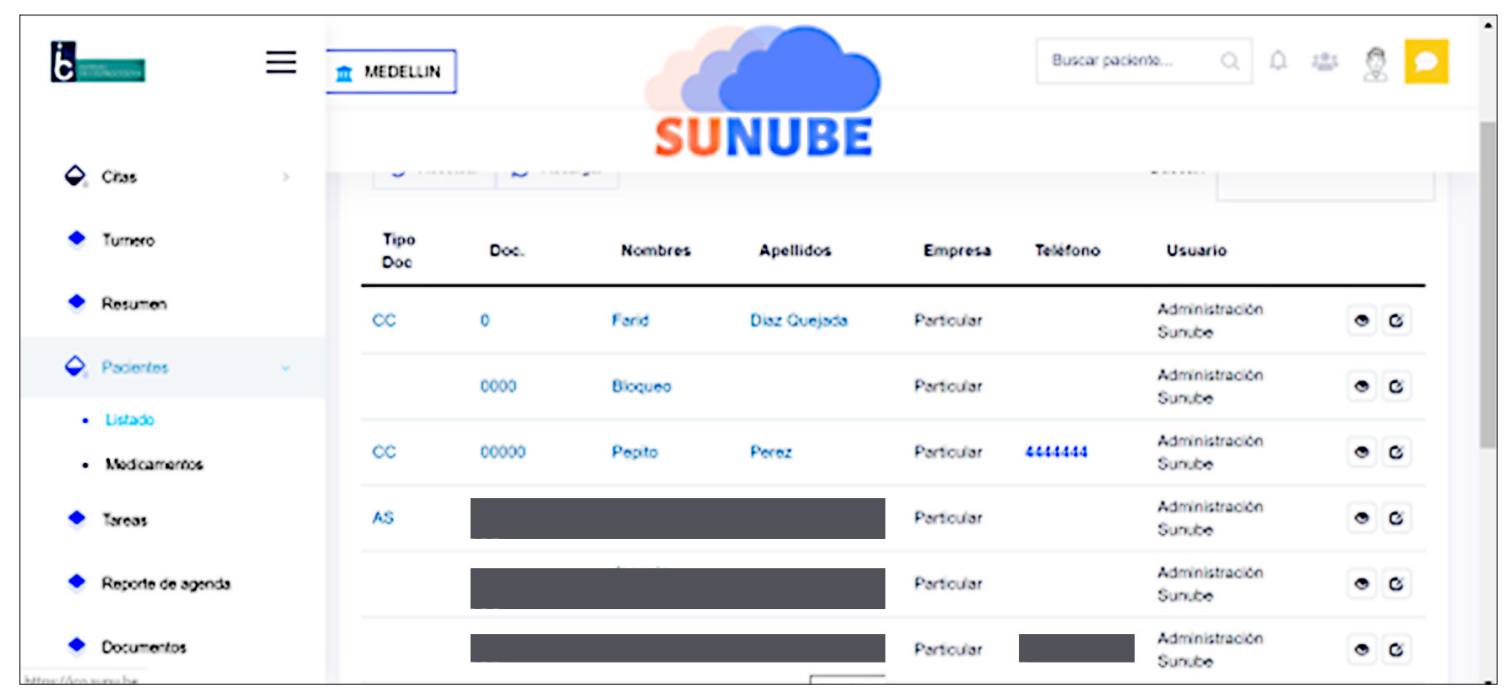

Figura 3. Portal página agenda electrónica SUNUBE. Fuente: Agenda electrónica SUNUBE.

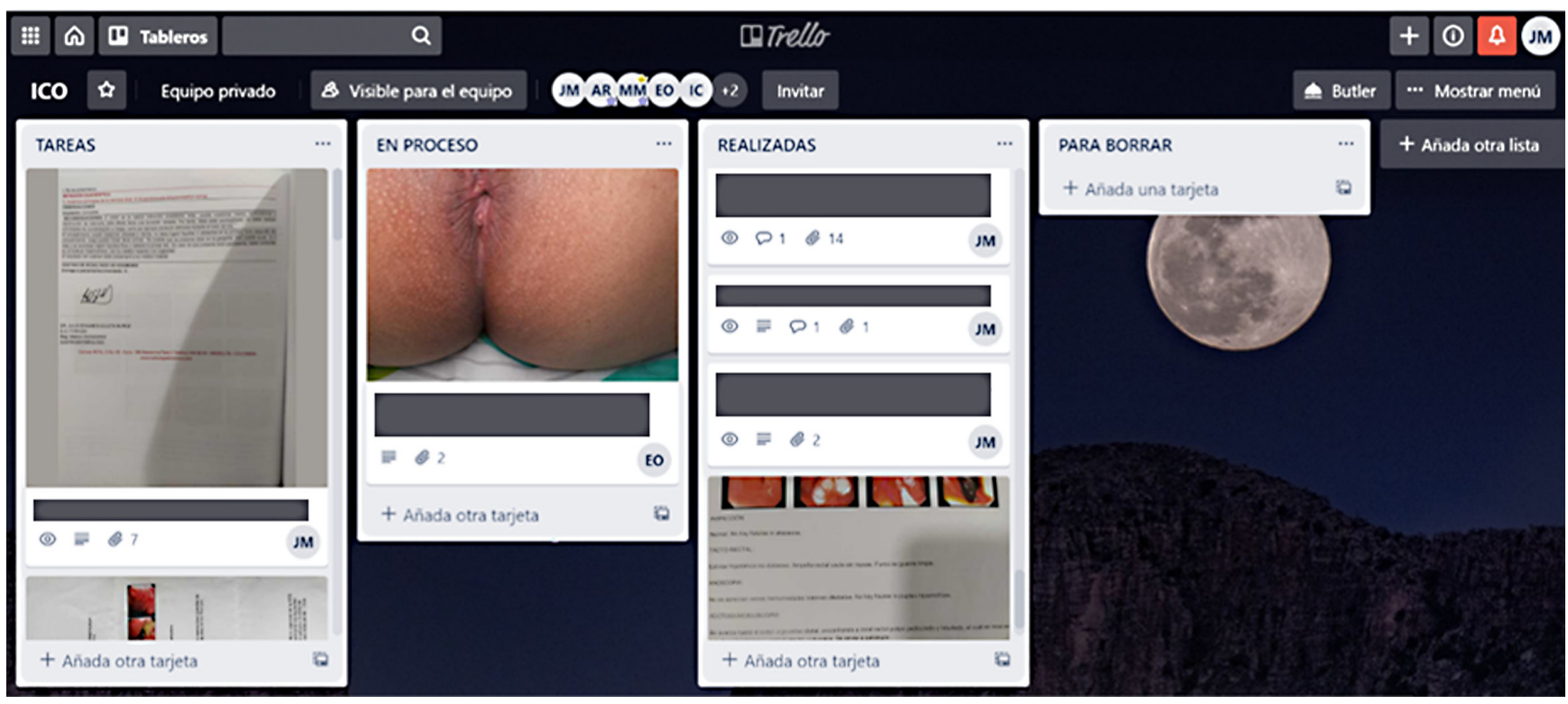

Figura 4. Portal página de la aplicación Trello®.

Mundial. La llamada gripe española, un nuevo virus de la gripe $\mathrm{A}\left(\mathrm{H}_{1} \mathrm{~N}_{1}\right)$, mató a más de 50 millones de personas, cifra superior al número de muertes asociadas con la mencionada confrontación bélica.

En los últimos 20 años se han presentado brotes epidémicos, como el síndrome respiratorio agudo severo (SARS, por sus siglas en inglés); en 2003, surgió en Asia con una afectación total de 8098 personas en todo el mundo (774 muertes); el síndrome respiratorio del Medio Oriente (MERS, por sus siglas en inglés), causado por un corona- virus detectado por primera vez en Arabia Saudita en 2012, que afectó a 1000 personas de 24 países y, al menos, 400 murieron en todo el mundo. Estos brotes afectaron zonas geográficas aisladas, lejanas de los grandes centros de poder económico y de comunicación, por tanto, fueron noticias secundarias en los medios (1).

Era cuestión de tiempo para que una pandemia afectara a la aldea global; gracias a los medios de comunicación, y dada la facilidad de transporte, es posible estar en cualquier país del mundo en menos de 24 horas. Ahora que el COVID-19 
está cercano y afecta directamente a médicos y a pacientes, emerge un pánico generalizado, más aún cuando nunca una infección fue amplificada por mil debido, también, a las redes sociales.

El mundo vive un fenómeno insospechado. Nunca se anticipó ni hubo planes para enfrentarlo, pero mientras se le hace frente a la pandemia del SARS-CoV-2 (COVID19), muchos sectores han visto la necesidad de reinventarse y acudir a la innovación para seguir adelante con sus rutinas y compromisos, y el sector salud no es la excepción. Tal y como señala Yuval Noah Harari, autor de Sapiens: de animales a dioses, "todas las emergencias aceleran procesos que ya venían en marcha”; y, según el politólogo Robert Kaplan, "las crisis como las guerras, ponen la historia a avanzar más rápido" (2).

El flujo caótico de pacientes en los departamentos de emergencias plantea un desafío único para el mantenimiento de la calidad de la atención, especialmente en tiempos de crisis y pandemias. El bienestar de la fuerza laboral de atención médica es la piedra angular de todo sistema de salud que aspire a funcionar adecuadamente. Como resultado de la actual pandemia, los proveedores de atención médica actúan bajo una enorme presión de carga de trabajo, junto con el aumento de los gastos totales en salud. La abrumadora carga del COVID-19 podría llevar al agotamiento y a la enfermedad del personal sanitario. Según la Comisión Nacional de Salud de China, más de 3300 trabajadores de la salud han sido infectados y, al menos 22, han muerto. En Colombia, al momento de escribir este artículo (el 19 de abril 2020), 4 médicos y un conductor de ambulancia han muerto y 169 servidores de la salud están infectados (Figura 5).

El triaje avanzado a través de la telemedicina puede convertir estos obstáculos en una oportunidad, proporcionar una vía para superar el flujo de pacientes, y reducir la carga de trabajo de los médicos. También puede minimizar el riesgo de exposición de los proveedores de atención médica. Debe recordarse que planificar con anticipación puede mejorar drásticamente las capacidades de gestión de crisis (3).

Una situación de pandemia en rápida evolución requiere comunicación interna y externa en tiempo real y la capacidad de adaptación rápida, mientras se mantiene la estabilidad del sistema, que, por lo general, se torna crítica (4).

La Circular del 17 de abril 2020 del Ministerio de Salud y Protección Social sobre la telesalud y la telemedicina para la prestación de servicios de salud en la pandemia por COVID-19, define lo siguiente:

- Telesalud: es el conjunto de actividades relacionadas con la salud, servicios y métodos, las cuales se llevan a cabo a distancia con la ayuda de las tecnologías de la información y telecomunicaciones. Incluye, entre otras, la telemedicina y la teleeducación en salud.

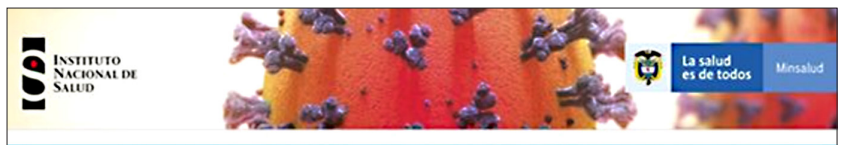

Datos abiertos de COVID-19 en personal de salud Colombia 2020, corte 19 abril 2020
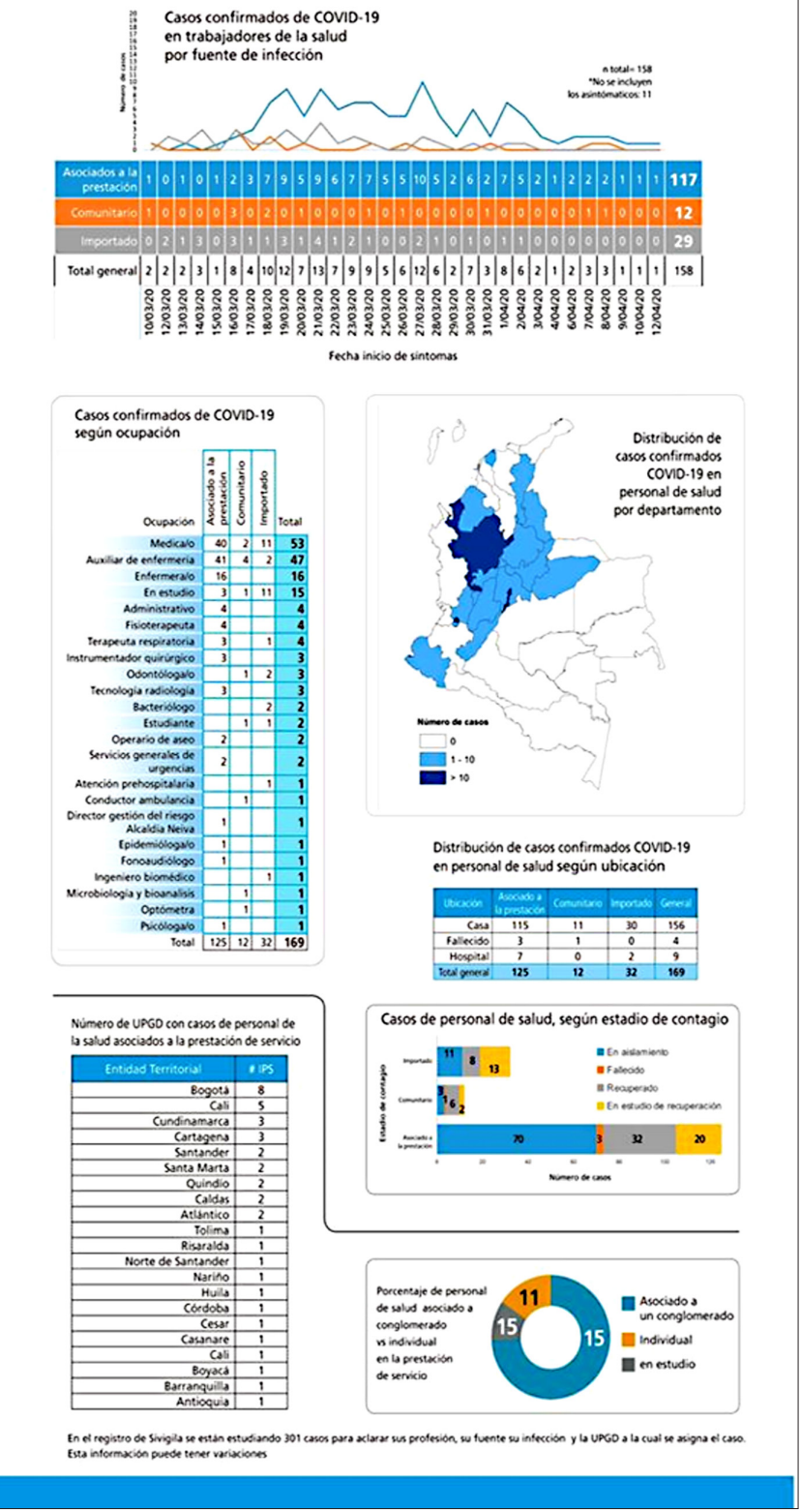

Figura 5. Datos del personal de la salud afectado por COVID-19 en Colombia (corte: 19 de abril 2020). Fuente: Instituto Nacional de Salud (INS).

- Teleorientación en salud: es el conjunto de acciones que se desarrollan a través de tecnologías de la información y comunicaciones para proporcionar al usuario información, consejería y asesoría en los componentes de 
promoción de la salud, prevención de la enfermedad, diagnóstico, tratamiento, rehabilitación y paliación. El teleorientador, en el marco de sus competencias, debe informar al usuario el alcance de la orientación y entregar una copia o resumen de la comunicación si el usuario lo solicita.

- Teleapoyo: se refiere al soporte solicitado por un profesional de la salud a otro profesional de la salud a través de tecnologías de la información y comunicaciones, en el marco del relacionamiento entre profesionales. Es responsabilidad de quien solicita el apoyo la conducta que determina para el usuario.

- Telemedicina: es la provisión de servicios de salud a distancia en los componentes de promoción, prevención, diagnóstico, tratamiento y rehabilitación por profesionales de la salud que utilizan tecnologías de la información y la comunicación, que les permiten intercambiar datos con el propósito de facilitar el acceso y la oportunidad en la prestación de servicios a la población que presenta limitaciones de oferta y de acceso a los servicios, o de ambos en su área geográfica.

- Telemedicina interactiva: es la relación a distancia utilizando tecnologías de información y comunicación mediante una herramienta de videollamada en tiempo real entre un profesional de la salud de un prestador y un usuario, para la prestación de servicios de salud en cualquiera de sus fases.

- Telemedicina no interactiva: es la relación a distancia utilizando tecnologías de información y comunicación mediante una comunicación asincrónica entre un profesional de la salud de un prestador y un usuario, para la provisión de un servicio de salud que no requiere respuesta inmediata.

- Telexperticia: es la relación a distancia con método de comunicación sincrónico o asincrónico para la provisión de servicios de salud en cualquiera de sus componentes, utilizando tecnologías de información y comunicación entre:

- Dos profesionales de la salud, uno de los cuales atiende presencialmente al usuario y otro atiende a distancia. El profesional que atiende presencialmente al usuario es responsable del tratamiento, de las decisiones y las recomendaciones entregadas al paciente; el que atiende a distancia es responsable de la calidad de la opinión que entrega y debe especificar las condiciones en las que se da dicha opinión, lo cual debe consignarse en la historia clínica.

- Personal de salud no profesional, esto es, técnico, tecnólogo o auxiliar, que atiende presencialmente al usuario y un profesional de la salud a distancia. El profesional que atiende a distancia será el responsable del tratamiento y de las recomendaciones que reciba el paciente; el personal de salud no profesional que atiende presencialmente al usuario será el responsable de las acciones realizadas en el ámbito de su competencia.

A nivel global, Malasia fue el primer país en tener una Ley de telemedicina en 1997, seguido por Francia y Brasil. Estas tres leyes difieren en su definición de telemedicina. La Ley de Malasia se refiere solo a la teleconsulta; la ley francesa se refiere a teleconsulta, tele-experticia, telemonitorización, teleasistencia y televigilancia; pero, en el caso de Brasil, la teleconsulta permite la consulta de médico a médico o de trabajador de salud a médico, y excluye explícitamente la consulta médico-paciente (5).

Como todo desafío definitorio en la historia humana, la aparición actual de COVID-19 viene con sus oportunidades únicas y sus propias soluciones innovadoras. Los avances tecnológicos brindan a la humanidad nuevas opciones, previamente no disponibles. Cuando el movimiento está restringido en todo el mundo y las ciudades enteras están en cuarentena, las poblaciones afectadas son susceptibles a mayores tensiones de la vida diaria, cargas económicas inesperadas, enfermedades transmisibles y no transmisibles, y trastornos de salud mental (6).

Aunque la solución definitiva para COVID-19 será multifacética, una vía importante que aún no se ha explorado por completo es aprovechar las tecnologías existentes para facilitar la prestación de atención óptima, y minimizar el riesgo de exposición directa de persona a persona. En este contexto, la telemedicina representa una opción atractiva, efectiva y asequible.

"La telemedicina está en etapas tempranas en Colombia y Latinoamérica. Menos de $1 \%$ de las consultas médicas son realizadas de manera remota actualmente. Sin embargo, tras el brote de coronavirus, la aceptación de la telemedicina ha aumentado más rápido de lo esperado y los Centros para el Control de Enfermedades (CDC) y la Organización Mundial de la Salud (OMS) están incentivando su uso para contener la expansión del virus" (2). Además, según Laura Gutiérrez, medicina fundadora de Doc Doc, una plataforma de doctores especialistas 24/7, "el marco legal pone a Colombia por delante de muchos países de Latinoamérica facilitando el acceso y la prestación de servicios de salud en cualquiera de sus fases (promoción, prevención, diagnóstico, tratamiento, rehabilitación y paliación)" (Figura 6) (2).

Una encuesta realizada este año con más de 2000 personas mostró que el $66 \%$ estaba de acuerdo con usar la telesalud, con un alto nivel de interés en todos los grupos de edad, incluido el $74 \%$ de encuestados de 18 a 44 años, y el $52 \%$ de 65 años y mayores. En total, el $25 \%$ informó que estaría dispuesto a cambiar a su médico de atención prima- 
ria por uno que ofreciera telesalud; pero solo el $8 \%$ reportó una experiencia real con una visita de video con un médico (7). Otra encuesta hecha en el 2016 por la Asociación Médica Americana (AMA) reportó que solo el 7,9\% de los gastroenterólogos reportan el uso de cualquier tipo de telesalud para interactuar con los pacientes.

Experiencias en este campo han sido tímidas y preliminares. Por ejemplo, el Centro de Ciencias de la Salud de la Universidad de Nuevo México (UNM) brinda apoyo especializado a proveedores de atención primaria para el tratamiento de la hepatitis $\mathrm{C}$ en poblaciones desatendidas mediante teleeducación y consulta de telemedicina. También se ha intentado mejorar el tratamiento de la EII a través de plataformas de telemonitorización que generan puntajes de riesgo en función de los síntomas informados por los pacientes, y alertan al médico tratante de posibles recaídas y descompensación (7).

Ha habido una experiencia preliminar en telemedicina desarrollada por un consenso para el empoderamiento y el autocuidado de pacientes con colitis ulcerativa (CU) leve a moderada, con el objetivo de que, rápidamente, estos puedan identificar a través de unas banderas rojas (sangre en las heces, aumento de frecuencia evacuatoria, etc.) que están frente a una exacerbación o brote de la enfermedad.

La iniciativa iSTART (i Support Therapy-Access to Rapid Treatment; en español: i Terapia de apoyo: el acceso al tratamiento rápido) brinda a los pacientes con CU de leve a moderado (seleccionados por su médico) la capacidad de autoevaluar la sintomatología de la CU, aumentar y optimizar la dosificación de la terapia de primera línea, e iniciar de manera autónoma con el tratamiento de segunda línea. Los médicos tratantes previamente deben seleccionar pacientes con ciertas características, tales como alto riesgo de recaída, acceso limitado a servicios de salud, proactivos en su autocuidado, o bajo riesgo de infección por Clostridium difficile.

Inicialmente, y bajo supervisión médica, se les indicará a los pacientes cómo optimizar su terapia de primera línea (mesalazina), una capacitación sobre cómo identificar un

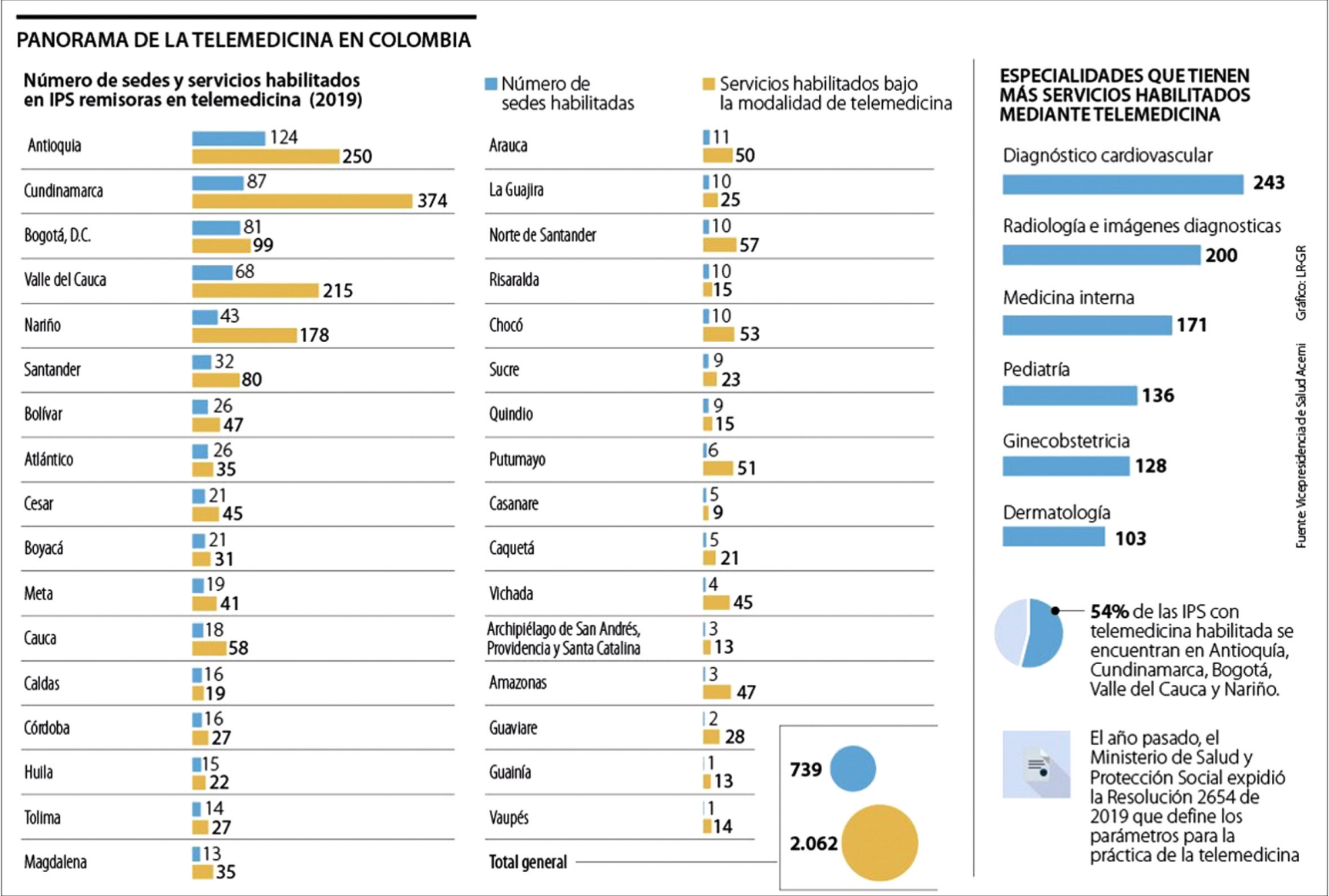

Figura 6. Panorama de la telemedicina en Colombia. Fuente: González X. [Internet]. Telemedicina, acceso más fácil a los servicios de salud por parte de todos los usuarios [24 de marzo de 2020]. Periódico La República. Disponible en: https://www.larepublica.co/especiales/101-buenas-ideas/ telemedicina-acceso-mas-facil-a-los-servicios-de-salud-por-parte-de-todos-los-usuarios-2981459 
brote sintomático, y la formulación con medicamentos de segunda línea (budesónida MMX) para su uso cuando el tratamiento de primera línea optimizado es insuficiente para mantener la remisión.

Aquí es donde la teleconsulta pudiera tener un papel muy importante. Cuando el paciente experimente o crea experimentar un brote, de manera autónoma empezará (de acuerdo con lo instruido) un curso corto de terapia de segunda línea, mientras contacta a su médico tratante. Lo anterior asegura que los pacientes no puedan automedicarse sin supervisión, pero sí que puedan, de manera autónoma y bajo supervisión médica a distancia, retomar el control de su enfermedad; esto, a la postre, redundará en menor morbilidad, mejoría en la calidad de vida y reducción de la congestión (y el riesgo de contagio) dentro de un servicio de urgencias, disminuyendo la carga asistencial clínica y la atención médica hospitalaria en el tratamiento de la CU leve a moderada (8).

La teleconsulta sirve, incluso, como método de triaje para COVID-19: la necesidad de limitar la exposición de pacientes potencialmente infecciosos y conservar el recurso hospitalario se debe considerar como prioridad. Si existe la sospecha, se debe preguntar y verificar mediante llamada telefónica (mejor videollamada) antes de que se presenten en persona, y evaluar de forma remota mediante un protocolo sencillo (9): "pedir que se tome la temperatura con un termómetro casero; documentar la apariencia general, observando si el paciente está enfermo, presenta diaforesis, palidez o rubicundez facial. Cálculo de la frecuencia respiratoria y observar la mecánica ventilatoria; verificar disnea de reposo y manera de hablar; uso de musculatura accesoria; presencia o ausencia de tos seca o productiva, etc. (4).

La teleconsulta es una forma segura y efectiva de evaluar casos sospechosos y guiar el diagnóstico y el tratamiento del paciente, minimizando el riesgo de transmisión de la enfermedad; en caso de una pandemia, los servicios de salud pueden colapsar a causa de una inundación de consultas que pudieran atenderse por medios virtuales (9). Además, las consultas programadas permiten la evaluación, la monitorización y el seguimiento de pacientes ambulatorios que no requieren una evaluación cara a cara; lógicamente, hay actividades que requieren la presencia física, y es allí donde se debe determinar cuándo la telepresencia es una opción y cuándo no (10).

Ante la realidad de que el país entraría en un confinamiento obligatorio de, al menos, 20 días, se citó una reunión urgente semipresencial (parte de los asistentes se conectaron por videollamada). La interacción se cumplió entre el personal administrativo, el personal médico, los funcionarios de la EPS SURA, el personal de soporte de telecomunicaciones y de sistemas.
Se acudió a la red y a la infraestructura física y tecnológica instalada de manera temprana pues, por sus características, es fácilmente moldeable y se ajusta a las necesidades del equipo. Se determinaron las mejores herramientas y se trazaron los flujos de trabajo con los estándares y prácticas vigentes. Específicamente, la privacidad del paciente, a quien debe protegerse del acceso no autorizado de un tercero durante la recopilación, transmisión y almacenamiento de información. El resultado de esta fase fue el diseño del protocolo de atención y de la hoja de ruta a seguir (11).

A los especialistas se les ofreció conseguir tarjetas SIM independientes para atender esta actividad con sus teléfonos celulares. Esta iniciativa surge de la estrategia adoptada por la Universidad de Washington, en Seattle, sitio que presentó en enero 2020 el primer caso de COVID-19 en Estados Unidos (4).

La telemedicina, en lo que respecta a la cirugía, puede servir entre otras para (12):

- Telerrobótica

- Telementoría

- Teleconsulta

- Telemedicina en el seguimiento posoperatorio

- Teleeducación

- Tecnología actual.

Para efectos de este artículo, se puso el foco en la teleconsulta y en la telemedicina en el seguimiento posquirúrgico.

Muchos centros de atención en el mundo, particularmente en las zonas rurales, carecen del personal o equipo capacitado para abordar la atención del paciente con patología que requiera evaluación quirúrgica. La aparición de la telemedicina y la teleconsulta ha planteado una oportunidad para los centros de atención que carecen de recursos, para ofrecer a sus pacientes una atención integral y multidisciplinaria.

Incluso, la EPS SURA tiene instituido el servicio de consulta virtual denominado "Concepto Virtual" entre el médico primario, especialista o supraespecialista, en el que los primeros, a través de la plataforma IPSA ${ }^{\circledR}$, registran los datos relevantes del paciente y realizan la consulta. El supraespecialista quirúrgico tiene 48 horas para responder a esta interconsulta y, luego de leer la historia clínica y los exámenes disponibles, entrega un concepto escrito de lo que, a su entender, debería hacerse con el paciente y autorizar su remisión para una evaluación presencial y prioritaria, si así lo amerita.

Desde el punto de vista posoperatorio, el seguimiento a los pacientes no es continuo después del alta hospitalaria, excepto por algunas visitas ambulatorias limitadas por tiempo y preprogramadas. Además, el período posterior a una intervención quirúrgica presenta un mayor riesgo de complicaciones debido a múltiples factores medioambienta- 
les, nutricionales, higiénicos, laborales y de autocuidado, los cuales, a menudo, se diagnostican con retraso, pues el conocimiento médico es ajeno a los pacientes y a sus familiares; de esta manera, les es difícil identificar signos tempranos de complicaciones. Como resultado, tiende a aumentar el riesgo de reingresos hospitalarios y de mortalidad $(12,13)$.

Una revisión sistemática investigó el papel actual de la telemedicina para facilitar la recuperación posoperatoria después del alta hospitalaria en los Estados Unidos. Según esta revisión, la telemedicina ofrece una amplia variedad de usos en la atención posoperatoria en diferentes especialidades que incluyen, entre otras, cirugía endocrina, ortopedia, otorrinolaringología, cirugía colorrectal, cirugía vascular, neurocirugía, trasplante, cirugía oncológica, urología y cirugía plástica (14).

La literatura registra referencias acerca del uso de la telemedicina para el cuidado periostomal, en el que una enfermera enterostomal examina el estoma de forma remota y asesora a una enfermera local sobre cómo cambiar las barreras, apósitos y demás materiales de curación; igualmente, entrega recomendaciones sobre cuidados de la piel, la alimentación y los signos de alarma frente a alta producción de efluente intestinal que pudiera producir desnutrición, deshidratación y quemaduras cutáneas; además, orienta sobre el uso de medicamentos formadores de bolo o espesantes de materia fecal (15).

La telemedicina se usó en un estudio con pacientes de cirugía vascular para controlar heridas y vigilar infecciones del sitio quirúrgico (ISQ). Para el efecto, los pacientes fotografiaron las incisiones y respondieron preguntas simples sobre la calidad del drenaje del líquido. El equipo quirúrgico responsable de las cirugías revisó e interpretó las fotos. Entre los 40 pacientes que completaron el protocolo del estudio, 7 de las 8 ISQ se detectaron mediante información visual y escrita de la aplicación. Una ISQ falso negativo se detectó en una visita temprana de seguimiento, y no hubo falsos positivos (16).

Para el sistema de salud, la telemedicina posoperatoria puede reducir los costos al liberar citas clínicas para otros usuarios, disminuir remisiones innecesarias y reducir la necesidad de reingreso hospitalario, mientras se incrementan los niveles de satisfacción por parte de pacientes y médicos $(12,13)$.

$\mathrm{Al}$ revisar diferentes experiencias de telemedicina a nivel mundial es posible extractar cuatro experiencias o modelos:

En Australia, por ejemplo, el énfasis en la videoconferencia excluye otros modelos de atención, usando tecnologías de monitorización e, incluso, combinando un conjunto de tecnologías complementarias como el correo electrónico y el audio. Para su aplicación, los prestadores de salud australianos cuentan con financiación exclusiva para esta actividad, conectividad confiable, población alfabetizada en tecnología y salud, y una comunidad con necesidad de acceso a esta modalidad, debido a la distancia, el aislamiento y las realidades locales (17).

El Departamento de Salud y Servicios Humanos de los Estados Unidos recientemente liberalizó las pautas de cumplimiento para enfrentar la pandemia COVID-19. Esa disposición permite utilizar una variedad de herramientas de comunicación que integran video, muchas de ellas gratuitas o de bajo costo; se incluyen Apple FaceTime, el chat de video de Facebook Messenger, el video de Google Hangouts y de Skype. Los pacientes requieren un teléfono inteligente, una tableta o computadora con audio y una cámara, así como un plan de datos; el hospital designará a una persona que actúe como "enlace tecnológico» al menos uno o dos días antes de la visita de telemedicina para proporcionar instrucciones de configuración y realizar una prueba de funcionamiento. Se ha flexibilizado tanto la regulación norteamericana a nivel federal que los usuarios pueden acceder al servicio de telemedicina, incluso a nivel interestatal; Medicare reembolsará las visitas de telemedicina y la Administración de Alimentos y Medicamentos (FDA, por sus siglas en inglés) permitirá la prescripción de sustancias controladas por telemedicina sin una evaluación previa en persona (18).

Brasil, país de dimensiones continentales y que cuenta con uno de los sistemas de salud pública más grandes y complejos del mundo, teóricamente garantiza el acceso universal y gratuito, así como la atención desde la cuna hasta la sepultura; pero, en la práctica, el cumplimiento de esta oferta está lejos de ser una realidad. En este contexto, la telesalud es una estrategia sólida para superar obstáculos como la mala distribución de los servicios de salud y la presencia desigual de profesionales en el territorio; esto causa largas filas de espera para la atención, referencias inadecuadas de pacientes, y riesgos en el desplazamiento de pacientes y profesionales. También son retos, afrontar la capacitación profesional limitada, las escasas inversiones gubernamentales sin garantía de educación continua, la pobre infraestructura local para la atención al paciente, y los crecientes índices y cinturones de pobreza.

En ese país, La Red de Telemedicina Universitaria (RUTE) fue creada por el Ministerio de Ciencia y Tecnología en colaboración con el Ministerio de Educación. Gracias a esta decisión, se ha implementado una infraestructura de videoconferencias para integrar el servicio de enseñanza y la investigación a través de Grupos de Interés Especial (SIG), y promover reuniones clínicas y discusiones técnico-científicas entre hospitales en una amplia gama de especialidades de salud. Por otro lado, además de que la legislación brasileña restringe las visitas médicas por videoconferencia, la infraestructura tecnológica es deficiente, lo que implica el acceso limitado a Internet en lugares remotos y los problemas de facturación y reembolso que representan barreras para una mayor expansión de la telesalud (17). 
En Canadá se consideran de vital importancia los medios digitales y virtuales para la prestación de la atención médica y la creación de capacidad dentro del sistema de salud, financiados con fondos públicos. Aspectos como la inteligencia artificial, el análisis avanzado y la tecnología blockchain han ingresado con fuerza en el panorama de la salud canadiense; sin embargo, los marcos normativos y reglamentarios no se corresponden, necesariamente, con la altura y los estándares de estos avances (17).

Revisar las experiencias de estos sistemas de telemedicina a nivel global permite extraer las siguientes conclusiones:

- Las tecnologías complejas no son esenciales: la teleconsulta, los mensajes de texto simples y el correo electrónico pueden ser suficientes.

- La infraestructura de las tecnologías de la comunicación es muy costosa. Crear un programa de telemedicina implica tiempo y no sucede durante la noche, ya que requiere fuentes de financiación, decisión política y planeación estratégica, además de garantizar cobertura a pesar de las grandes distancias, para llegar a sitios remotos con bajo nivel socioeconómico.

- Los fondos gubernamentales no garantizan una cobertura generalizada, espacio en el que la empresa privada debe entrar a cofinanciar diferentes emprendimientos.

- Su mayor cualidad es superar las barreras de la distancia, pero puede ser muy útil en áreas urbanas al proveer atención primaria y secundaria de manera eficiente, rompiendo las dificultades de acceso y descongestionando los servicios hospitalarios.

Con todo y sus virtudes, la teleconsulta también tiene aspectos negativos. Por ejemplo:

- Altera los modelos tradicionales de atención, por lo tanto, la relación cercana médico-paciente se podría debilitar o, incluso, perderse.

- Se considera que, dentro del acto médico, para llegar a un diagnostico el $60 \%$ corresponde a la anamnesis; mientras el $40 \%$ restante corresponde al examen físico e, incluso, al lenguaje no verbal manifestado por el paciente y percibido por el médico cuando se está frente a frente o, en otras palabras, "el ojo clínico". En esta especialidad dicho examen es fundamental para definir, en diferentes ocasiones, entre una conducta médica o quirúrgica, y para determinar cuál será el mejor abordaje, si se opta por esta última.

- La telesalud exige emplearse en forma ética, balanceada y controlada; de lo contrario, puede prestarse para ampliar la percepción sobre la llamada "deshumanización" de la medicina. Se corre el riesgo de favorecer su control y manejo por medio de herramientas tecnológicas (big data e inteligencia artificial) para interactuar con el paciente $y$, a través de algoritmos, definir conductas clínicas.
- La videollamada es lo más cercano a tener un contacto físico entre médico y paciente; para llevarla a cabo se requieren: un teléfono inteligente; tableta o computadora con audio y una cámara; un plan de datos; y una plataforma o aplicación informática. La realidad colombiana, a diferencia de otras latitudes, dibuja un país pobre en el que la gran mayoría de la población no dispone de estos elementos, dada la baja capacidad adquisitiva; de paso, una de las poblaciones potencialmente beneficiaria, los adultos mayores tampoco tienen los conocimientos para su correcta implementación, lo que también podría ocurrir en otros grupos poblacionales con baja escolaridad.

Durante la actual pandemia del COVID-19, la OMS ha pedido a las naciones que aumenten su preparación, planteando las siguientes tres prioridades:

- Proteger a los trabajadores de la salud.

- Junto con las comunidades, se debe trabajar activamente en identificar e implementar formas de protección de las personas con mayor riesgo de enfermedad grave; en particular, los ancianos y las personas con afecciones de salud preexistentes.

- En tercer lugar, la comunidad global debe proteger a los países más vulnerables, haciendo todo lo posible para contener la epidemia y minimizar su propagación.

Dentro del contexto de las prioridades de la OMS, los servicios de telemedicina están perfectamente diseñados y acondicionados para ayudar a alcanzar los objetivos que se derivan de estas tres prioridades:

1. La telemedicina protege activamente a los trabajadores de la salud al reducir las interacciones no agudas entre pacientes y personal de salud, lo que minimiza el riesgo de transmisión del COVID-19.

2. La teleconsulta ayudará a las comunidades a proteger a las personas de alto riesgo (los ancianos y las personas con condiciones de salud concomitantes) al reducir su exposición en hospitales y centros con alta carga infecciosa potencial.

3. Los países o regiones con gran cantidad de personal y recursos de atención médica podrán ayudar a los países o regiones con acceso limitado, proporcionando personal y recursos (6).

Los planes de salud, los empleadores, los sistemas hospitalarios y los medios de comunicación deben trabajar para superar estas barreras reduciendo costos, educando y concientizando a las personas sobre la teleconsulta, una alternativa efectiva y más segura en las circunstancias actuales, y ofreciendo instrucciones paso a paso sobre cómo funciona y cómo se puede acceder a ella. Igualmente, es necesario llegar 
a una negociación con los entes gubernamentales y privados para que el pago o el reembolso por esta actividad sea adecuado para los profesionales de la salud involucrados (1).

Aunque no se han elaborado encuestas de satisfacción ni otros estudios sólidos con este propósito, nuestra percepción es que, bajo las circunstancias especiales que atraviesa hoy la humanidad, los pacientes valoran y agradecen de manera especial que su médico los llame, los escuche y los atienda tratando de resolver, hasta donde sea posible, su situación de salud. Así, evitan salir de sus domicilios, donde están resguardados y cómodos, sin exponerse a un posible contagio; esto, en última instancia, podría conducir a mejores resultados en calidad y cobertura de la atención médica. Los proveedores de los servicios de salud también se benefician con mejores niveles de satisfacción y retención del paciente, pues sus usuarios reciben un servicio especializado, digno, adecuado y oportuno, con mayor eficiencia en el flujo de trabajo y con la capacidad de cumplir con las expectativas de servicio a sus usuarios

\section{CONCLUSIÓN}

La telemedicina se constituye en una gran oportunidad para mejorar los sistemas de apoyo a la salud. El desarrollo y la implementación de la infraestructura de telecomunicaciones implica un enorme costo; por lo tanto, son prioritarios en el mundo entero los programas de salud virtuales junto con una legislación que impulse la adopción de la telemedicina como una posibilidad para corregir la pobre relación médico-paciente en cualquier sociedad. Este tipo de iniciativas especiales, unidas al uso eficiente de la teleeducación, proporcionarán un desarrollo integral que se traducirá en mayor cobertura, sin sacrificar la calidad en la atención.

Una vez superada la pandemia actual, y cuando el virus se vuelva endémico, la humanidad sobrevivirá y seguirá el curso de su historia. Y, aunque parece un lugar común, vale insistir en que nunca nada será igual. Dentro de los cambios que transformarán definitivamente la salud, la telemedicina ocupará un lugar destacado y, en ese posicionamiento, la ampliación y el perfeccionamiento del servicio de teleconsulta desempeñarán un papel determinante. La teleconsulta, aún percibida como una opción en determinados contextos sociales y geográficos complejos, llegó para quedarse y proporcionar a los pacientes una atención más conveniente y costo-efectiva. De esta manera, la humanidad estará preparada para afrontar su nueva realidad, y, sobre todo, para la próxima pandemia que, inevitablemente, llegará en el futuro.

\section{AGRADECIMIENTOS}

En la experiencia que se describe participaron los miembros del equipo profesional del Instituto de Coloproctología
ICO S.A.S. De alguna manera, este estudio también lleva la firma de su entrega, dedicación y amor por la medicina: Adriana Ruiz, Luis Francisco Miranda, Ernel Alberto Duque, Mario Muñoz, Cindy Vanessa Meneses, Luisa Fernanda Morales, Valeria Martínez, Elsy Alejandra López, Ailín Caterín Zuluaga, Angélica María Acosta e Ingrid Viviana Rodríguez.

\section{REFERENCIAS}

1. Portnoy J, Waller M, Elliott T. Telemedicine in the Era of COVID-19. J Allergy Clin Immunol Pract. 2020;22132198. https://doi.org/10.1016/j.jaip.2020.03.008

2. González X. [Internet]. Telemedicina, acceso más fácil a los servicios de salud por parte de todos los usuarios [24 de marzo de 2020]. Periódico La República. Disponible en: https://www.larepublica.co/especiales/101-buenas-ideas/ telemedicina-acceso-mas-facil-a-los-servicios-de-salud-porparte-de-todos-los-usuarios-2981459

3. Moazzamia B, Razavi-Khorasania N, Dooghaie Moghadama A, et al. COVID-19 and telemedicine: Immediate action required for maintaining healthcare providers well-being. Journal of Clinical Virology. 2020;126:104345. https://doi. org/10.1016/j.jcv.2020.104345

4. Grange ES, Neil EJ, Stoffel M. Responding to COVID19: The UW Medicine Information Technology Services Experience. Appl Clin Inform. 2020;11(2):265-275. https://doi.org/10.1055/s-0040-1709715

5. Mars M. Medicolegal, ethical, and regulatory guidelines pertaining to telehealth. En: Gogia S, editor. Fundamentals of Telemedicine and Telehealth. Cambridge: Elsevier; 2020. p. 297-303. https://doi.org/10.1016/B978-0-12-8143094.00013-6

6. Chauhan V, Arquilla B, Gaiwankar S, et al. Novel Coronavirus (COVID-19): Leveraging Telemedicine to Optimize Care While Minimizing Exposures and Viral Transmission. Journal of Emergencies Trauma and Shock. 2020;13(1):20-24.

7. Lee T, Kim L. Telemedicine in Gastroenterology: A Value-Added Service for Patients. Clin Gastroenterol Hepatol. 2020;18(3):530-533. https://doi.org/10.1016/j. cgh.2019.12.005

8. Danese S, Banerjee R, Cummings JrF, et al. Consensus recommendations for patient-centered therapy in mild-to-moderate ulcerative colitis: the i Support Therapy-Access to Rapid Treatment (iSTART) approach. Intest Res. 2018;16(4):522528. https://doi.org/10.5217/ir.2018.00073

9. Hollander JE, Carr BG. Virtually Perfect? Telemedicine for Covid-19. N Engl J Med. 2020;382(18):1679-1681. https://doi.org/10.1056/NEJMp2003539

10. PanAmerican Health Organization (PAHO) [Internet]. Teleconsultations during a pandemic - Factsheet. [Abril de 2020]. Disponible: https://www.paho.org/ish/images/ docs/covid-19-teleconsultations-en.pdf?ua $=1$ 
11. Greenhalgh T, Choon Huat Koh G, Car J. Covid-19: a remote assessment in primary care. BMJ. 2020;368:m1182. https://doi.org/10.1136/bmj.m1182

12. Huang EY, Knight S, Roginski Guetter G, et al. Telemedicine and telementoring in the surgical specialties: A narrative review. Am J Surg. 2019;218(4):760-766. https://doi. org/10.1016/j.amjsurg.2019.07.018

13. Cremades M, Ferret G, Pares D, et al. Telemedicine to follow patients in a general surgery department. A randomized controlled trial. Am J Surg. 2020. https://doi.org/10.1016/j. amjsurg.2020.03.023

14. Gunter RL, Chouinard S, Fernandes-Taylor S, et al. Current use of telemedicine for post-discharge surgical care: a systematic review. J Am Coll Surg. 2016;222(5):915-927. https://doi.org/10.1016/j.jamcollsurg.2016.01.062
15. Bogen EM, Augestad KM, Patel HR, Lindsetmo R-O. Telementoring in education of laparoscopic surgeons: an emerging technology. World J Gastrointest Endosc. 2014;6(5):148155. https://doi.org/10.4253/wjge.v6.i5.148

16. Gunter RL, Fernandes-Taylor S, Rahman S, et al. Feasibility of an image-based mobile health protocol for postoperative wound monitoring. J Am Coll Surg. 2018;226(3):277-286. https://doi.org/10.1016/j.jamcollsurg.2017.12.013

17. Pearce C. Worldwide initiatives. En: Gogia S, editor. Fundamentals of Telemedicine and Telehealth. Cambridge: Elsevier; 2020. p. 331-342. https://doi.org/10.1016/B9780-12-814309-4.00015-X

18. Calton B, Abedini N, Fratkin M. Telemedicine in the Time of Coronavirus. J Pain Symptom Manage. 2020. https://doi. org/10.1016/j.jpainsymman.2020.03.019 